\title{
Electric current activated sintering (ECAS) of undoped and titanium-doped BiFeO3 bulk ceramics with homogeneous microstructure
}

M.S. Bernardo ${ }^{1,2}$, T. Jardiel ${ }^{1}$, A.C. Caballero ${ }^{1}$, M. Bram³ ${ }^{3}$ J. González-Julian ${ }^{3}$, M. Peiteado ${ }^{1,2}$

\author{
${ }^{1}$ Department of Electroceramics, Instituto de Cerámica y Vidrio - CSIC, Kelsen 5, Campus de \\ Cantoblanco, 28049, Madrid \\ ${ }^{2}$ POEMMA-CEMDATIC, ETSI Telecomunicación (UPM), 28040 Madrid, Spain \\ ${ }^{3}$ Forschungszentrum Jülich GmbH, Institute of Energy and Climate Research: Materials Synthesis and \\ Processing (IEK-1), 52425 Jülich, Germany
}

Undoped and $\mathrm{Ti}^{4+}$-doped $\mathrm{BiFeO}_{3}$ ceramics have been consolidated by applying a flash sintering methodology under different electrical conditions. The DC experiments evidence a strong localization of the current flow which, empowered by the semiconductor behaviour of $\mathrm{BiFeO}_{3}$, leads to a dramatic lack of microstructural and compositional homogeneity. The situation drastically changes in the presence of an alternate field; a more gradual FAST event is instead produced which allows the attainment of an unprecedented homogeneity through the whole sintered cylindrical compacts. The incorporation of titanium to the $\mathrm{BiFeO}_{3}$ nominal composition slows down the overall diffusivity of the system which delays the onset of the flash, but again a remarkable homogeneity is obtained for the AC experiments, this time resulting in the particular nanostructure typically observed in titanium doped $\mathrm{BiFeO}_{3}$ materials. Thus, it is shown that undoped and $\mathrm{Ti}^{4+}$-doped $\mathrm{BiFeO}_{3}$ bulk ceramics with a functional micro or nanostructure can be attained by a flash-sintering methodology, at much lower temperatures and times than by a conventional solid-state sintering.

\section{Introduction}

Magnetoelectric materials with coupled ferroelectricity and ferromagnetism within the same phase are earning increased interest in the last decade due to their potential applications in spintronics, memory devices and so on [1-3]. Among the very few known materials of this kind, $\mathrm{BiFeO}_{3}$ has been postulated as the most promising one because its 
high phase transition temperatures may provide a magnetoelectric response even at room temperature [4-6]. However, in practice, $\mathrm{BiFeO}_{3}$ materials show several limitations that hamper their practical applications. Particularly detrimental is the high level of dielectric losses which are generally ascribed to the existence of defects such as oxygen vacancies, in the crystalline structure [7] and/or to the presence of secondary phases [8], very difficult to avoid in the processing of these materials $[9,10]$. Besides, due to the longrange order magnetic structure of this compound, the net magnetic moment is annihilated for nanocrystals larger than $64 \mathrm{~nm}$ [11]. Several studies have revealed that the magnetic spin cycloid can be frustrated either by decreasing the nanocrystal size or by distorting the crystalline structure by doping with certain ions [12-14]. In any case, a strict control of the processing parameters is required to obtain materials with the desired composition and microstructure that might lead to a suitable magnetoelectric response. As a matter of fact, $\mathrm{BiFeO}_{3}$-based ceramic powders with suitable phase purity and tailored particle size may be prepared by meticulously controlling the synthesis conditions and/or by using alternative synthesis methodologies; but even in these cases the situation is usually further reverted during the consolidation of the synthesized powders to obtain dense materials $[15,16]$. The reasons for the difficulties in sintering $\mathrm{BiFeO}_{3}$ lies in the fact that, besides the general requirement of controlling equilibrium between densification and grain coarsening, additional problems related with the synthesis complexity and the low thermal stability of these compounds are to be faced [17]. Furthermore, the high temperatures needed for sintering may also result in a loss of stoichiometry as a consequence of bismuth volatilization, leading to a higher concentration of oxygen vacancies and eventually resulting in an increased electrical conductivity.

Visibly, the possibility of consolidating $\mathrm{BiFeO}_{3}$ ceramics by alternative low-sintering techniques would be greatly welcome to avoid the above the mentioned difficulties. Electric current activated/assisted sintering (ECAS) techniques are gaining increasing attention for the processing of advanced ceramic materials due to their multiple advantages compared to the conventional sintering, which usually requires higher temperatures and much longer holding times [18-20]. As reviewed by Yu et al. [21], flash sintering has been applied to consolidate a wide range of materials types such as ionic conductors, semiconductors, etc. during the last decade. Strictly speaking, there are several ECAS techniques, which are usually classified depending on the electrical parameters and boundary conditions. A clear differentiation between various ECAS 
techniques is given by the peak volumetric power dissipation against discharge time [21], which reflects two different mechanisms of field-assisted sintering: the gradual enhancement in sintering has been denoted as FAST while the nearly stepwise sintering has been called flash sintering. The flash sintering of nanostructured $\mathrm{BiFeO}_{3}$ has been recently reported for the first time by Perez-Maqueda et al. [22, 23]: working with the characteristic dog-bone shaped samples for hanging flash-sintering experiments, these authors show that the field-assisted sintering can be helpful in preventing the bismuth losses and retaining the $\mathrm{BiFeO}_{3}$ phase. As they observe, the structural/compositional quality of the sintered samples depends on the applied DC field and the current limit through the sample, and this is to be due to the localisation of the current flow. The dependence on the electric conditions on the current localisation was also stressed by M'Peko et al. [24] in their earlier work on the field-assisted sintering of $\mathrm{BaTiO}_{3}$ ceramics. These other authors proposed that the strength of the electric field and the current limits on the specimen may determine the success of the ECAS process giving rise to two different processing regions: the so-called "safe-region", where sintering is uniform, and the "fail-region", where the current flow in the sample becomes localised leading to heterogeneous microstructures. Actually, it is well admitted that the field-assisted sintering under direct currents (DC) may result in an asymmetrical microstructural development [21, 25-27], this eventually leading to very different grain sizes and densities throughout the sintered specimen. This effect could be avoided with an alternate current (AC) electric field. In this view, the first experiments considering the equivalence of AC and DC electric field sintering have been recently reported for the flash sintering of YSZ materials [28, 29] but, to the best of our knowledge, a similar analysis on the field-assisted sintering of $\mathrm{BiFeO}_{3}$-based materials under an alternate current (AC) has not been described yet.

On the other hand, in addition to the alternative processing strategies, one of the most accepted approaches to obtain BFO-based materials with an adequate electric and magnetic response is doping [30, 31]. In this sense, many different ions have been tested to substitute the bismuth or iron atoms at the A or B positions of the perovskite structure, respectively [32-35]. Among the multiple possibilities, during the last years it has been found that $\mathrm{Ti}^{4+}$-doping (or co-doping) results particularly interesting since it may decrease the electrical conductivity of the BFO-based materials and may even provide a net magnetic response [7, 36-41]. Consequently, the effect of $\mathrm{Ti}^{4+}$-doping on the structure, 
microstructure and properties of $\mathrm{BiFeO}_{3}$ materials has been extensively studied. Our previous work [40] revealed that this dopant (usually added in substitution of the iron atoms) produces a peculiar effect in the microstructure development due to the dopant tendency to segregate; this leads to a decreased grain boundary conductivity that controls the macroscopic electric response of the material, as well as to the formation of crystalline domains with a mean size of about $50 \mathrm{~nm}$ (in which the $\mathrm{Ti}^{4+}$ ions are mainly located at the boundary regions) that frustrates the magnetic-spin cycloid resulting in a net magnetic response. Thus, it can be expected that both the microstructural and electrical effects caused by this dopant will also strongly affect the flash sintering of the $\mathrm{BiFeO}_{3}$ materials.

So in this frame, the aim of this contribution is to study the effect of the electric current conditions on the compositional and microstructural homogeneity of the DC and AC field-assisted sintering of undoped and titanium-doped $\mathrm{BiFeO}_{3}$. The high sensitivity of this particular system to the experimental sintering conditions (leading to significant changes in the crystalline phases and microstructure) may serve as an indicator of the mechanisms underlying the flash sintering. This will be analysed on cylindrical samples whose simple geometry and area-to-volume ratio can simplify the interpretation of the presumed phenomenon of current localization.

\section{Experimental procedures}

Ceramic powders with $\mathrm{BiFeO}_{3}(\mathrm{BFO})$ and $\mathrm{BiFe}_{0.95} \mathrm{Ti}_{0.05} \mathrm{O}_{3}$ (BFTO) nominal compositions were prepared by a conventional solid-state procedure under the optimized experimental conditions described elsewhere [15, 34]. This includes a first step of synthesis (prereaction) in which the corresponding stoichiometric amounts of the starting oxides were mixed and calcined at $750{ }^{\circ} \mathrm{C}$ for the undoped composition and $800{ }^{\circ} \mathrm{C}$ for the Ti-dopedone. These green pre-reacted powders were milled and uniaxially pressed at $15 \mathrm{kN}$ to shape cylindrical green bodies with diameter of $8 \mathrm{~mm}$ and length of $10 \mathrm{~mm}$. The geometrically measured density of the BFO and BFTO green bodies were 5.3 and 5.0 $\mathrm{g} / \mathrm{cm}^{3}$; this is $60 \%$ and $64 \%$ of the $\mathrm{BiFeO}_{3}$ theoretical density $\left(6.346 \mathrm{~g} / \mathrm{cm}^{3}\right)$, respectively.

Samples were sintered in an air atmosphere in a modified sinter-forging setup, composed of a vertically split furnace fixed on a mechanical testing machine [42, 43]. Height and diameter of the samples were measured in situ by a laser system (162-100; BETA Laser Mike, Dayton. $\mathrm{OH}$ ) every second with a resolution of $0.5 \mathrm{~mm}$ in order to calculate the 
shrinkage during the experiments. The specimens were placed between two platinum electrodes, which were electrically insulated from the testing machine by two alumina disks. A minimal pressure of around 0.15 MPa was applied to ensure the contact between the samples and the electrodes during the whole test. Specimens were heated at $10{ }^{\circ} \mathrm{C} / \mathrm{min}$ up to the selected sintering temperature and maintained isothermally for 30 minutes. The electrodes were connected to a power supply DC/AC voltage (ACS-Power-Source; HBS Electronic GmbH, Brühl, Germany). DC experiments were conducted applying electric fields of 30,40 and $50 \mathrm{~V} / \mathrm{cm}$, whereas the $\mathrm{AC}$ tests were all done at $40 \mathrm{~V} / \mathrm{cm}$ and applying frequencies of $50 \mathrm{~Hz}, 500 \mathrm{~Hz}$ and $1 \mathrm{kHz}$. No external current limits were imposed for any of these experiments and the current actually flowing through the sample was measured every second in the course of the tests. The power dissipated within the specimens $\left(\mathrm{W} / \mathrm{cm}^{3}\right)$ was calculated from the product of the electric field and the current density flowing through the sample. Several experiments were carried out per composition and condition to average the results.

The final densities of all the specimens were calculated by Archimedes Method in water and were used to calibrate the sintering curves. In all cases densities around 90-95\% of the theoretical density of pure $\mathrm{BiFeO}_{3}$ were attained. Sintered samples were photographed using a NIKON D-3500 digital camera. For microstructural analysis samples were cut by their half in the direction parallel to the applied electric field. Polished cross-section surfaces were observed in a Field-Emission Scanning Electron Microscope (Hitachi S4700) supplied with an energy-dispersive spectroscopy (EDS) microanalysis probe. Grain boundaries were revealed on polished surfaces by chemically etching the samples with diluted $\mathrm{HCl}$. Grain-size measurements were evaluated from FESEM micrographs by an image processing and analysis program (Leica). Structural analysis was carried out through X-ray diffraction (XRD) on a Bruker Advance diffractometer using $\mathrm{Cu} \mathrm{K} \alpha$ radiation, both on the polished cross-section surfaces as well as on powdered ceramic pieces ground in a tungsten mortar (these pieces were selected from specific regions of the samples, as described in the next section).

\section{Results and discussion}


As mentioned in the experimental section, the DC tests were conducted applying electric fields of 50, 40, and $30 \mathrm{~V} / \mathrm{cm}$. However, as it will be further discussed, the microstructural characterization of these samples reveals that $40 \mathrm{~V} / \mathrm{cm}$ is the minimum field strength to be applied in order to produce a field-assisted sintering. Similarly, the AC tests were run under different frequencies; although some differences were indeed observed as a function of the applied frequency, these were not enough to produce relevant microstructural changes in the sintered material. Accordingly, on what follows, the discussion of the DC experiments will be related to the $40 \mathrm{~V} / \mathrm{cm}$ and $50 \mathrm{~V} / \mathrm{cm}$ specimens, and the discussion of the AC results will be focused on the $50 \mathrm{~Hz}(40 \mathrm{~V} / \mathrm{cm})$ sintered samples.

\subsection{Flash sintering of undoped $\mathrm{BiFeO}_{3}$.}

The plots of the axial shrinkage and the power dissipation registered during the sintering cycles of undoped BFO ceramics under different electric conditions are displayed in

Figure 1. Under a direct current electric field of $50 \mathrm{~V} / \mathrm{cm}$, the current starts flowing through the undoped BFO materials when the furnace reaches temperatures about $600{ }^{\circ} \mathrm{C}$ (heating rate of $10^{\circ} \mathrm{C} / \mathrm{min}$ ). Then at $650^{\circ} \mathrm{C}$, a power dissipation spike peak is produced (Figure 1.a), the current density reaches a maximum value (limit) of ca. $33 \mathrm{~mA} / \mathrm{mm}^{2}$, and this is accompanied by the abrupt shrinkage of the material (Figure 1.b), which in less than 2 minutes experiments an axial shrinkage of around 15\%. Thus, the concurrence of sintering with the maxima in the volumetric power dissipation against discharge time indicates the onset of the flash [21]. The observed values of the flash event at $50 \mathrm{~V} / \mathrm{cm}$ DC are quite similar to those recently reported by Maqueda and co-workers [22], who perceived an onset of ca. $625^{\circ} \mathrm{C}$ for a current limit of $35 \mathrm{~mA} / \mathrm{mm}^{2}$. In their experiments these authors start from a mixture of the constituent oxides, not from a pre-reacted powder, so our results again indicate that despite the differences in the processing history, the effect of the DC electric field is strong enough to produce a significant lowering in the furnace temperature required to sinter the $\mathrm{BiFeO}_{3}$ ceramics (typically requiring temperatures above $800{ }^{\circ} \mathrm{C}$ to get dense materials [34]).

Under a DC electric field of lower magnitude, namely $40 \mathrm{~V} / \mathrm{cm}$, the material also starts sintering at $600{ }^{\circ} \mathrm{C}$. However, in this case the shrinkage takes longer times to be completed, about 10 minutes (see Figure 1.b). Besides, the maximum in the volumetric 
power dissipation occurs after the sample has sintered to a certain degree in a process, which is produced gradually. The current limit is also achieved gradually, this time reaching a maximum value of ca. $24 \mathrm{~mA} / \mathrm{mm}^{2}$ (visibly smaller than that of the sample sintered under $50 \mathrm{~V} / \mathrm{cm}$ DC, $33 \mathrm{~mA} / \mathrm{mm}^{2}$ ). According to M'Peko et al. [24], such gradual profiles of the curves indicate that, under these "softer" electric conditions the sintering is being produced by a FAST mechanism rather than by a flash event.

Lastly, undoped BFO ceramics were also sintered under an AC electric field of $40 \mathrm{~V} / \mathrm{cm}$. Figure 1 evidences that under these electric conditions, the BFO specimens start sintering at similar temperatures that under a DC field of the same magnitude. The sintering also occurs in a gradual fashion, again indicating a FAST process more than a flash event, and the only difference with the analogue DC tests is the current density limit through the sample during the cycles, which in this case reaches a maximum value of ca. $9 \mathrm{~mA} / \mathrm{mm}^{2}$ (effective current), the smaller one of all these experiments.
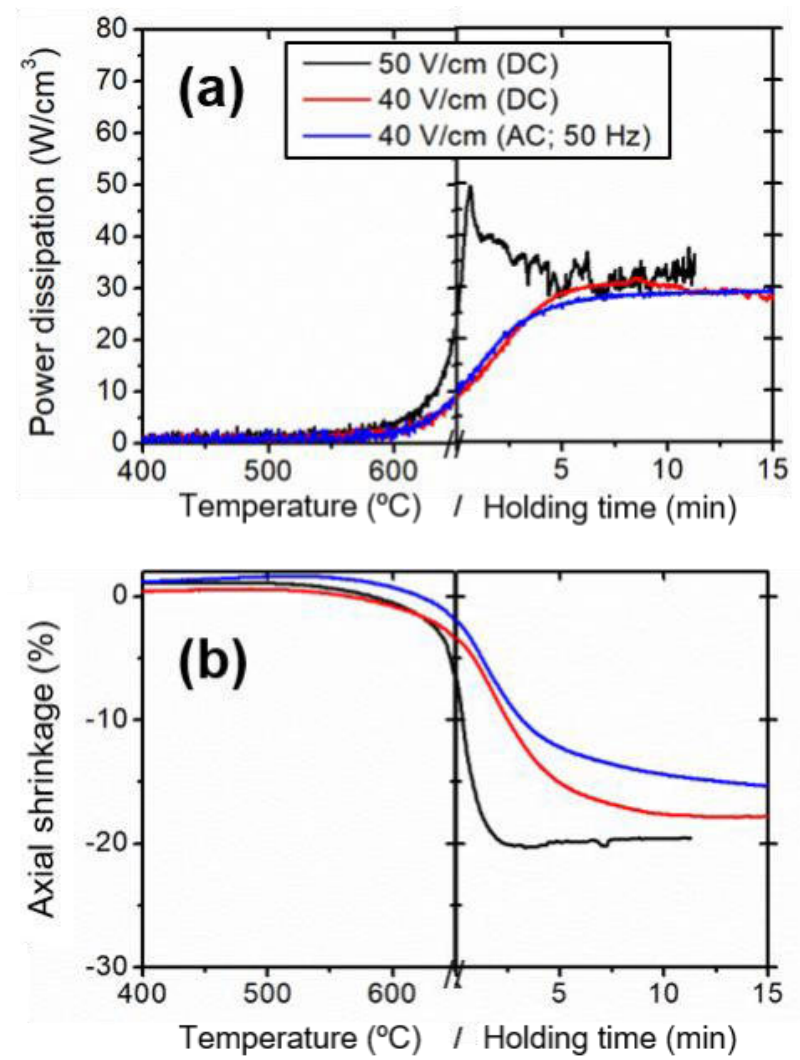
Figure 1. Power dissipation (a) and axial shrinkage (b), for the undoped BFO materials sintered under different electrical conditions.

Figure 2.a shows the external appearance of the specimens sintered under the above described conditions. As observed, the three samples initially looked well densified and relatively homogeneous, although some noticeable deficiencies can be perceived too. At the top of the samples, i.e. close to the region in contact with the upper electrode (the cathode), a reddish to brown coloured area of ca. $0.5 \mathrm{~mm}$ in thickness is observed in all cases; this is indeed the typical appearance of green poorly sintered bismuth ferrite. In the DC treated pellets, this un-densified area goes together with the presence of a physical damage in their top surface, namely a grey spot of about 1-2 $\mathrm{mm}$ in diameter with a melting-like appearance (see the inset in Figure 2.a); such defect, which is not observed in the AC treated material, probably results from a major current localisation at that point [24]. More differences emerge at the bottom of the pellets, in the region close to the lower electrode (the anode). For the DC samples this bottom zone appears partially melted, especially for the higher electric field of $50 \mathrm{~V} / \mathrm{cm}$, and actually the pellets remained slightly stuck to the Pt electrode after the sintering cycles. That melted feature is however not observed in the AC sample, which instead shows the same not-sintered reddish region that in its top side, evidencing a more symmetrical scenario in this last experiment. Following the external inspection, the samples were cut in half (bisected) in the direction parallel to the electric field and here is where the effect of the different electrical conditions applied to the consolidation step of the materials become further evident. As depicted in the photographs in Figure 2.b, regions with distinct appearance can be observed within the samples, pointing out to the existence of several areas with different chemical compositions and/or structures. For instance, when sintering under a DC electric field of $50 \mathrm{~V} / \mathrm{cm}$, four different regions can be identified. The first region or region I at the top part of the sample, would comprise the reddish brown covering of the top surface plus a whitish area which slightly extends towards the sides of the pellet; as mentioned that is the typical appearance of a poorly sintered $\mathrm{BiFeO}_{3}$ pre-reacted power which has seen not enough temperature to properly densify. The second region, or region II, with a dark-grey tonality, spreads all over the lateral sides of the cylindrical sample. However, the region II does not reach the central part of the sample, where a new different area is clearly observed, region III, that vastly comprises the centre of the specimen: it shows a 
predominant light-grey (or whitish grey) tone with certain metallic shine, and as observed it also involves some macroscopic holes of large dimensions (ca. $1 \mathrm{~mm}$ in diameter) surrounded by a reddish environment. Lastly, at the bottom part of the specimen, another region can be distinguished, region IV, this one showing a grey tonality and big holes corresponding to the melted material. These four regions can be again identified on the sample sintered under a DC field of $40 \mathrm{~V} / \mathrm{cm}$, although their extension results different in this case; in particular, the so-called region II is now visibly larger, and this time no holes nor metallic spots can be perceived in the central region III. But once again the most noticeable differences emerge when looking to the sample sintered under an AC electric field. As depicted in Figure 2.b, the cross-section surface of this specimen is now practically fully covered by the dark grey tonality corresponding to region II, and the only macroscopic defects that can be perceived (a flat stripe at the middle of the sample and the concentric circles on its edges) could be better related to the shaping stage (the uniaxial pressing of the cylindrical pellets) and/or the pressure being applied to the pellets during sinter-forging setup, than to the effect of the electrical field itself. In other words, this photograph evidences that in this AC treated specimen an extraordinarily homogeneous situation is successfully produced which extends macroscopically through the whole material, something that is not so easily attained when working under flash or FAST sintering conditions.

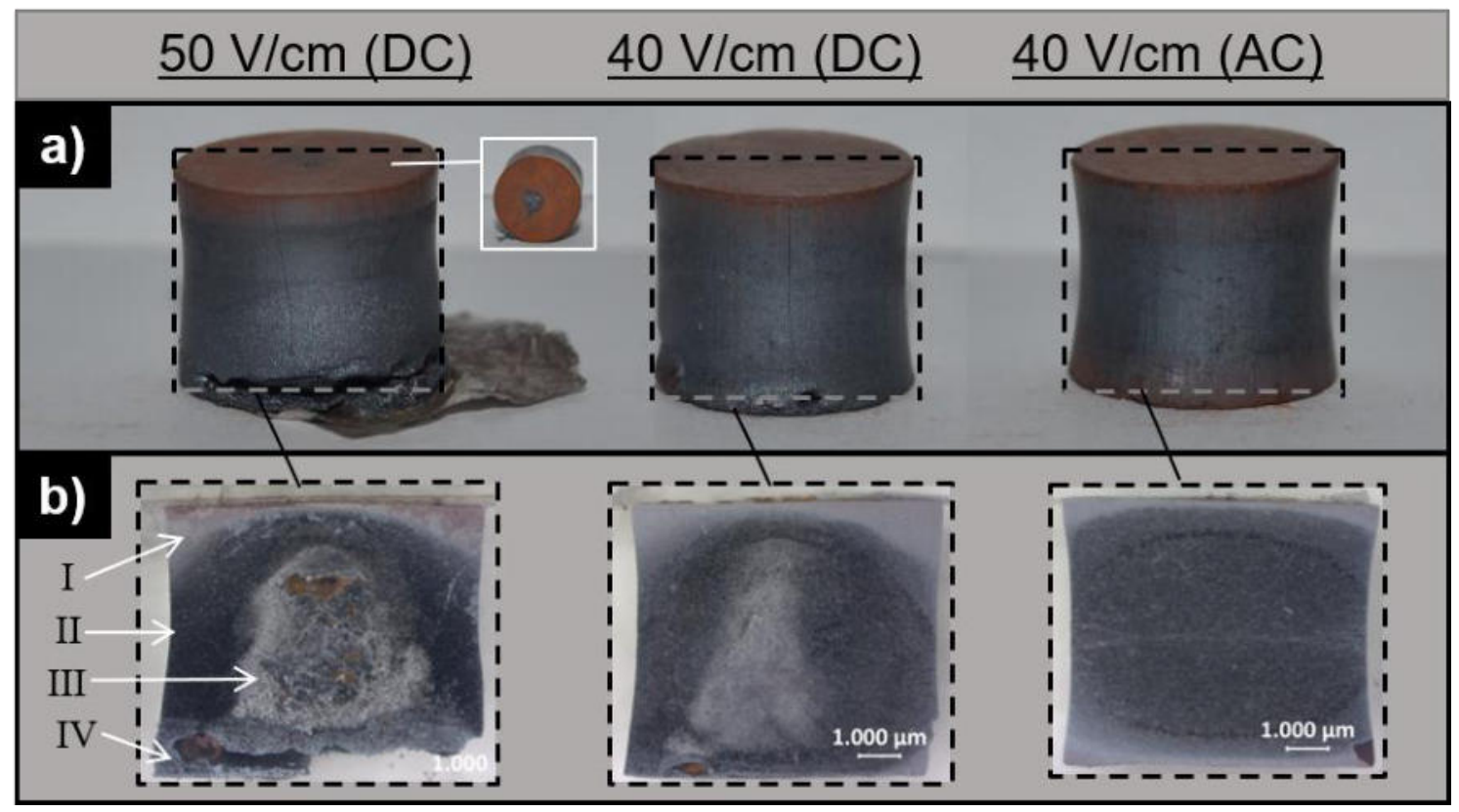

Figure 2. Photographs of the BFO materials after sintering under different electrical conditions taken on the entire as-sintered specimens (a) or on the cross-section polished 
surface (b); the regions with different appearance (see the text for further description) are indicated in the first photograph.

The specific characteristics of the different regions described in Figure 2 were further learned by means of XRD and FESEM analyses. For all the tested samples, this characterization first confirmed the complete absence of a sintered microstructure in region I, with almost no changes in the XRD pattern from that of the starting powder (not shown here). Figure 3 shows the results of the microstructural studies performed on the three remaining regions of the BFO sample sintered under a DC field of $50 \mathrm{~V} / \mathrm{cm}$. Figure 3.a shows the X-ray diffractogram mainly obtained from the surface of region II, evidencing the presence of two crystalline phases: the main $\mathrm{BiFeO}_{3}$ perovskite phase (ICDD No. 01-071-2494), and the secondary $\mathrm{Bi}_{25} \mathrm{FeO}_{39}$ bismuth-rich sillenite-type phase (ICDD No. 01-077-0865). The XRD pattern depicted in Figure 3.b mainly corresponding to region III shows the presence of two additional phases: $\mathrm{Bi}_{2} \mathrm{Fe}_{4} \mathrm{O}_{9}$ iron-rich mullite-type phase (ICDD No. 00-025-0090) and metallic Bi (ICDD No. 00-044-1246); and something similar occurs with the diffractogram in Figure 3.c, taken from region IV, which depicts the presence of an additional $\mathrm{Fe}_{2} \mathrm{O}_{3}$ phase (ICDD No. 00-033-0664). These compositional differences are further evinced in the corresponding FESEM images for this DC treated sample. On one hand, Figure 3.d shows the microstructure which dominates the region II, consisting on large $\mathrm{BiFeO}_{3}$ grains of about 20 microns in size which are all surrounded by a secondary Bi-rich phase (the $\mathrm{Bi}_{25} \mathrm{FeO}_{39}$ sillenite phase, according to semiquantitative EDS analyses). This particular microstructure is consistent with a liquidphase assisted sintering process, similar to that observed in conventionally sintered BFO materials, but at higher temperatures: around $850{ }^{\circ} \mathrm{C}$ or above [44]. In other words, the observed microstructure indicates that under the applied DC field $(50 \mathrm{~V} / \mathrm{cm})$ the temperature inside the region II has reached a considerable higher temperature than the one truly applied in the furnace, which as mentioned was set at $650{ }^{\circ} \mathrm{C}$ in these experiments. The increase of the specimen temperature much above the furnace temperature is usually observed in the ECAS experiments and it is attributed to a Joule heating effect [37] and/or to the occurrence of the flash event itself [18]. On the other hand, the microstructure corresponding to region III (Figure 3.e) also shows a multiphase scenario in which a visible number of $\mathrm{Bi}_{2} \mathrm{Fe}_{4} \mathrm{O}_{9}$ mullite grains are now dispersed on a sort of $\mathrm{BiFeO}_{3}$ matrix. No clear evidence of the sillenite interconnected frame observed in 
region II is here perceived; instead, a myriad of light grey coloured, Bi-rich zones are observed through the whole region that can be associated with the $\mathrm{Bi}^{0}$ metallic phase detected by XRD. Actually, the presence of the latter can be explained by a volatilization plus reduction process of the sillenite phase; like pure $\mathrm{Bi}_{2} \mathrm{O}_{3}$, the sillenite structure has a high vapour pressure and will easily melt with an uncontrolled increase of the temperature, like the one occurring here due to the current localization during the flash sintering. In the regions close to the external surface of the sample, this melt will simply volatilize to the atmosphere, but in the internal regions a reductive atmosphere will be created which combined with the rapid increase in the sample temperature (the flash) will eventually result in the condensation of $\mathrm{Bi}^{0}$ [45]. Finally, the microstructure corresponding to region IV is shown in Figure 3.f. This region was partially melted (Figure 2.a.) and, hence, its microstructure evidences a largely decomposed material in which $\mathrm{Fe}_{2} \mathrm{O}_{3}$ coexists as practically the main phase with the $\mathrm{BiFeO}_{3}$ perovskite phase. Again, the unrestrained increase in temperature provoked by the flash event is behind this microstructural context, this time pointing out to the partial decomposition of the $\mathrm{BiFeO}_{3}$ perovskite phase: according to previous studies on the $\mathrm{Bi}_{2} \mathrm{O}_{3}-\mathrm{Fe}_{2} \mathrm{O}_{3}$ solid-state reaction mechanisms, once the $\mathrm{BiFeO}_{3}$ perovskite phase is completely formed from its constituent oxides, the $\mathrm{Fe}_{2} \mathrm{O}_{3}$ phase can just be released (and then detected) when the peritectic decomposition of the $\mathrm{BiFeO}_{3}$ is produced, something which starts to occur at temperatures above $930^{\circ} \mathrm{C}[10]$. 

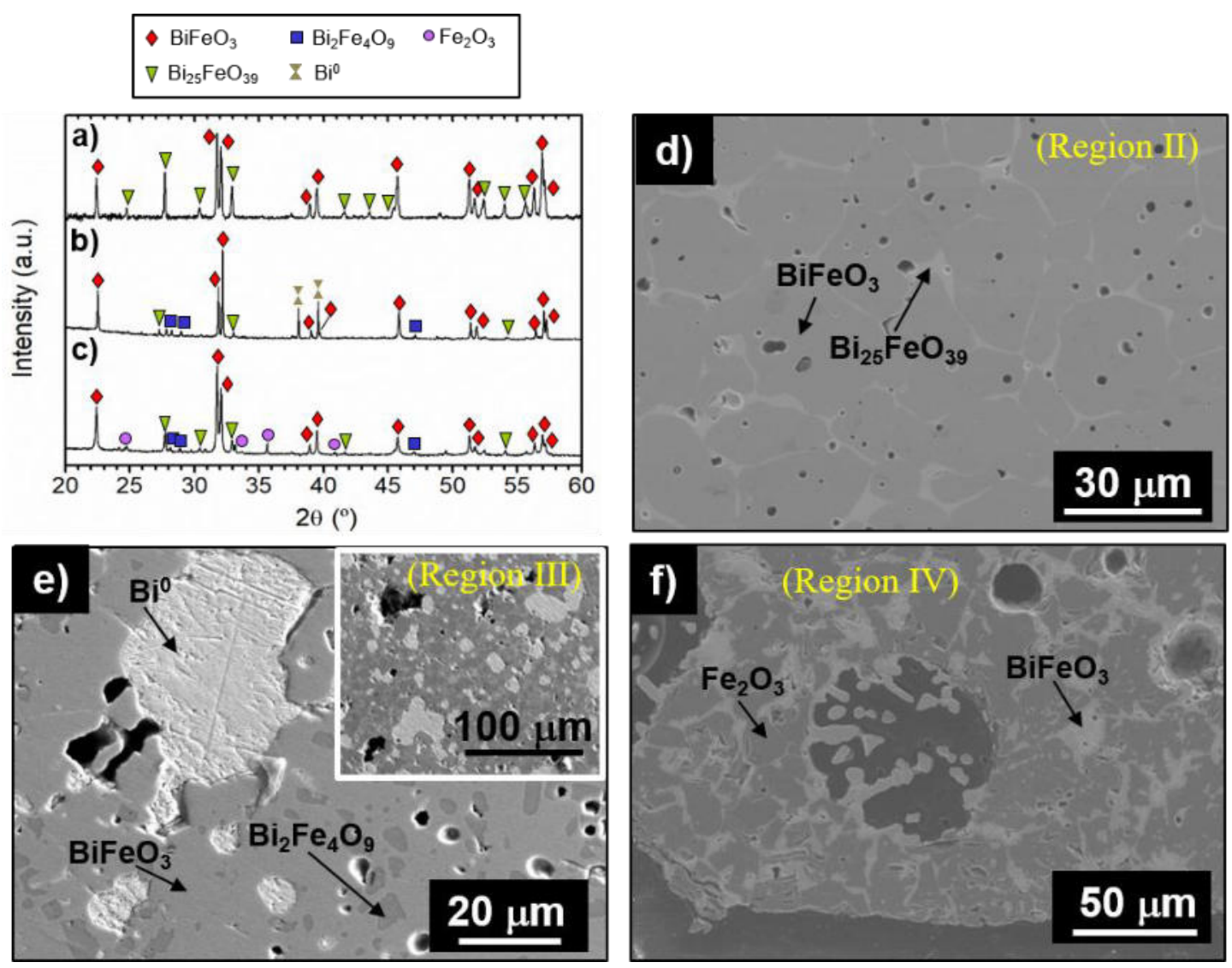

Figure 3. XRD and FESEM characterization of the BFO specimen sintered under a DC electric field of $50 \mathrm{~V} / \mathrm{cm}$, and corresponding to the different regions identified in Figure 2 (see explanation in the text). Compounds marked in the FESEM images have been identified with the aid of EDS analyses.

So, at this point we have seen that under a DC field of $50 \mathrm{~V} / \mathrm{cm}$, an asymmetrical microstructural development is produced in the BFO material in which up to four regions with different microstructure and composition can be observed all throughout the specimen. As expected the flash event must be producing an increase in the temperature inside the material, but under these initial conditions this is far from being homogeneous leading to the existence of thermal gradients within the sample as a consequence of the current localisation. As mentioned, this localisation upon the injection of carriers is not unusual when working with direct currents, [19-21] but it is probably more stressed in the $\mathrm{BiFeO}_{3}$ composition due to its semiconductor behaviour. In fact, as shown in Figure 2, the material is almost unreacted in the vicinity of the cathode (upper electrode) and partially melted in the anode (bottom electrode), evidencing the localisation track of the carriers. One feasible way to reduce the localisation of the current flow is to decrease the magnitude of the applied DC field [24], something that we tested with our BFO samples 
by reducing the field strength from $50 \mathrm{~V} / \mathrm{cm}$ to $40 \mathrm{~V} / \mathrm{cm}$. As described, this first produces a transition from a flash event to a more gradual FAST sintering mechanism (Figure 1), which also leads to a certain microstructural improvement: as shown in Figure 2.a, the melting of the material in the vicinity of the bottom electrode is clearly less obvious in the $40 \mathrm{~V} / \mathrm{cm}$ treated specimen. The snapshot of the bisected specimens in Figure 2.b also shows that the distribution of the different regions inside the $40 \mathrm{~V} / \mathrm{cm}$ sample is prone to improve; the extension of the regions in which the perovskite phase decomposes is significantly smaller and this goes together with an appreciable increase in the volume of region II (initially the finest one as seen in the XRD/FESEM analyses) and a significant reduction in the secondary phase proportion over all the regions. Thus, initially we could take these facts as good indicative of a successful flash sintering process, at least in this region II. Even though, we still have an asymmetrical structural/compositional distribution in the sample and the material is far from being homogenous at the macroscopic level, meaning that the formation of thermal gradients, i.e. the current localisation, is still not prevented. A further decrease in the applied DC field was expected to be useful in this sense. However, as mentioned before, our tests with $30 \mathrm{~V} / \mathrm{cm}$ DC yielded no field-assisted sintering at all (too low power for these cylindrical units). So finally, the only way to achieve an effective microstructural improvement was through the AC experiments. Under such electrical conditions, specifically $40 \mathrm{~V} / \mathrm{cm}$ and $50 \mathrm{~Hz}$, we have already seen that the system also evolves through a FAST mechanism (Figure 1.a), which leads to a symmetrical external appearance (Figure 2.a) and eventually to an extremely homogeneous microstructure that extends macroscopically inside the whole material (Figure 2.b). Figure 4 now shows the XRD/FESEM characterization of that microstructure. The obtained XRD pattern evidences an almost pure $\mathrm{BiFeO}_{3}$ perovskite phase and barely some traces of the $\mathrm{Bi}_{25} \mathrm{FeO}_{39}$ sillenite phase could be detected in the analyses (Figure 4.a). The scanning electron micrographs taken from the chemically etched polished cross-section surface (Figure 4.b), show a homogeneous microstructure which is mainly composed by well-faceted $\mathrm{BiFeO}_{3}$ grains with an average size in between 3-5 microns. In fact, this smaller grain size indicates a significant reduction of the amount of $\mathrm{Bi}_{25} \mathrm{FeO}_{39}$ liquid phase during sintering compared to what was obtained when the material was sintered under a DC field. In consonance, the microstructural characterisation on the -not etched- polished surface (see inset in Figure 4.b) shows that, under this last conditions, the proportion of the unwelcomed secondary phases is drastically reduced; as previously described, the finest region for the sample treated under 
a $50 \mathrm{~V} / \mathrm{cm}$ DC field consisted of $\mathrm{BiFeO}_{3}$ grains clearly bigger in size $(20 \mu \mathrm{m})$ and all surrounded by the sillenite phase (see and compare Figure 4.b with Figure 3.d). But moreover, the microstructure obtained under the alternate current greatly resembles that of BFO ceramics conventionally sintered at $800{ }^{\circ} \mathrm{C}$ during 2 hours, indicating that this particular flash sintering conditions were successful for obtaining a functional BFO microstructure at lower temperature and time. The observed differences can be first ascribed to the lower effective current under AC conditions, leading to a lower Joule heating effect and consequently to a lower temperature in the specimen. But besides, the larger contribution of the grain interior to the conductivity of the material under AC fields may aid in preventing the current localisation, which eventually results in a much more homogeneous current distribution, in the absence of large thermal gradients along the material and, hence, in the avoidance of macroscopic defects.
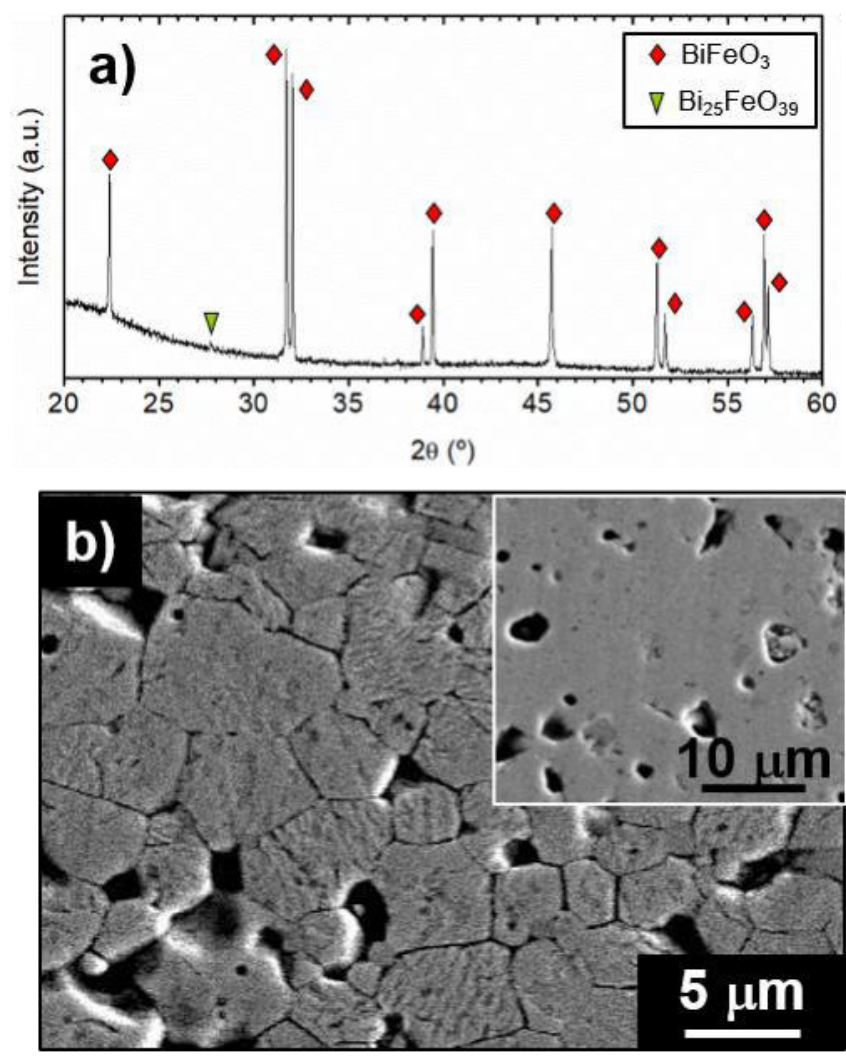

Figure 4. X-ray diffractograms (a) and scanning electron micrograph (b) on the chemically etched polished cross-section surface of a BFO sample sintered under an AC electric field of $40 \mathrm{~V} / \mathrm{cm}$; the inset shows the non-etched polished surface of a similar sample (note that some holes on the surface are originated during the polishing process). 


\subsection{Flash sintering of $\mathrm{Ti}^{4+}$-doped $\mathrm{BiFeO}_{3}$ (BFTO).}

Ti-doped $\mathrm{BiFeO}_{3}$ (BFTO) ceramics were sintered under the same electric conditions than the undoped material and, similar to what happened with the BFO undoped composition, the best results in terms of microstructural homogeneity were attained for the AC treated samples. In this view Figure 5 displays the evolution of the axial shrinkage and the power dissipation for the BFTO sample during its sintering under an AC field of $40 \mathrm{~V} / \mathrm{cm}$. Two different behaviours can be already observed from the evolution of the BFO specimen. On one hand the onset of the flash event is now produced at $700{ }^{\circ} \mathrm{C}$, i.e. $50^{\circ} \mathrm{C}$ above than for the undoped composition (although still considerably lower than for a conventionally sintered BFTO: above $825^{\circ} \mathrm{C}$ [34]). Such increase must be attributed to the presence of $\mathrm{Ti}^{4+}$ whose incorporation to the $\mathrm{BiFeO}_{3}$ nominal composition is known to delay the diffusion processes in the system and, hence, increases the sintering temperature required to get fully dense materials [34, 40, 44]. On the other hand, these plots in Figure 5 also show that both the bulk shrinkage and the maximum in the power dissipation are both reached in a shorter time (less than 2 minutes; almost 10 minutes for the analogue BFO specimen) indicating that here we are again more close to a flash event. Under these conditions the maximum in the current flow now reaches a value of $16 \mathrm{~mA} / \mathrm{mm}^{2}$.

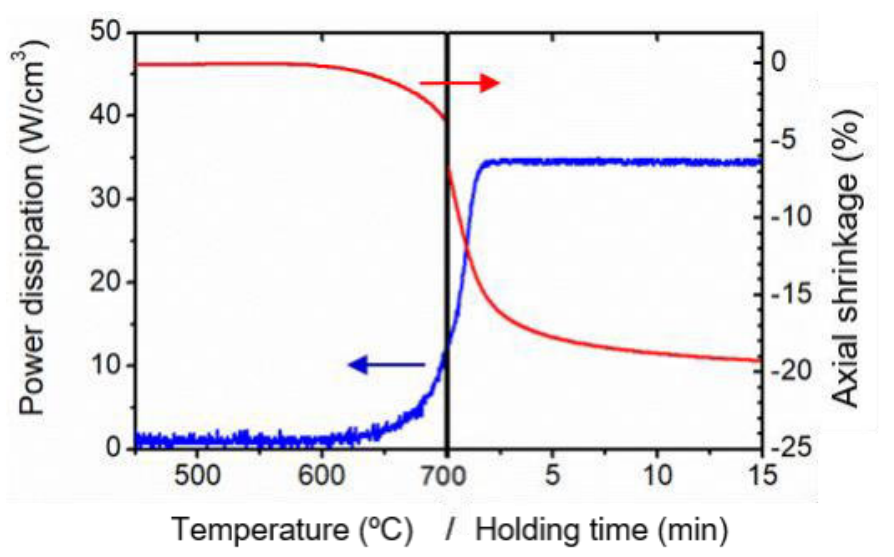

Figure 5. Power dissipation and axial shrinkage for the BFTO material sintered under a $40 \mathrm{~V} / \mathrm{cm}$ AC electric field.

The structural/compositional characterization of the BFTO sample sintered under these $40 \mathrm{~V} / \mathrm{cm}$ AC conditions is summarized in Figure 6. The corresponding photographs of the as-sintered specimen, Figure 6.a, evidence that both the external appearance and the 
internal microstructural distribution of the cylindrical pellet exhibit the same symmetrical features that were observed for the undoped material, so again indicating that the localization of the current flow can be initially prevented under the alternate currents. The corresponding XRD pattern in Figure 6.b shows broadened diffraction peaks of the main $\mathrm{BiFeO}_{3}$ perovskite phase, together with a certain amount of the $\mathrm{Bi}_{25} \mathrm{FeO}_{39}$ and $\mathrm{Bi}_{2} \mathrm{Fe}_{4} \mathrm{O}_{9}$ secondary phases, whose proportion relatively increases compared to the undoped material sintered under same electric conditions. Even though, this does not prevent for attaining a homogeneous functional microstructure like the one depicted in Figure 6.c, which mainly consists of uniformly distributed $\mathrm{BiFeO}_{3}$ grains that in this case just reach an average size of 200-400 nanometres. As mentioned, such a particular nanostructure is actually quite similar to the one that can be observed when the BFTO nominal composition is obtained through a conventional sintering process [27,29], indicating that the $\mathrm{AC}$ flash sintering methodology can be also applied to successfully produce $\mathrm{BiFeO}_{3}$ materials with a high level microstructural homogeneity at the nanoscale level. 

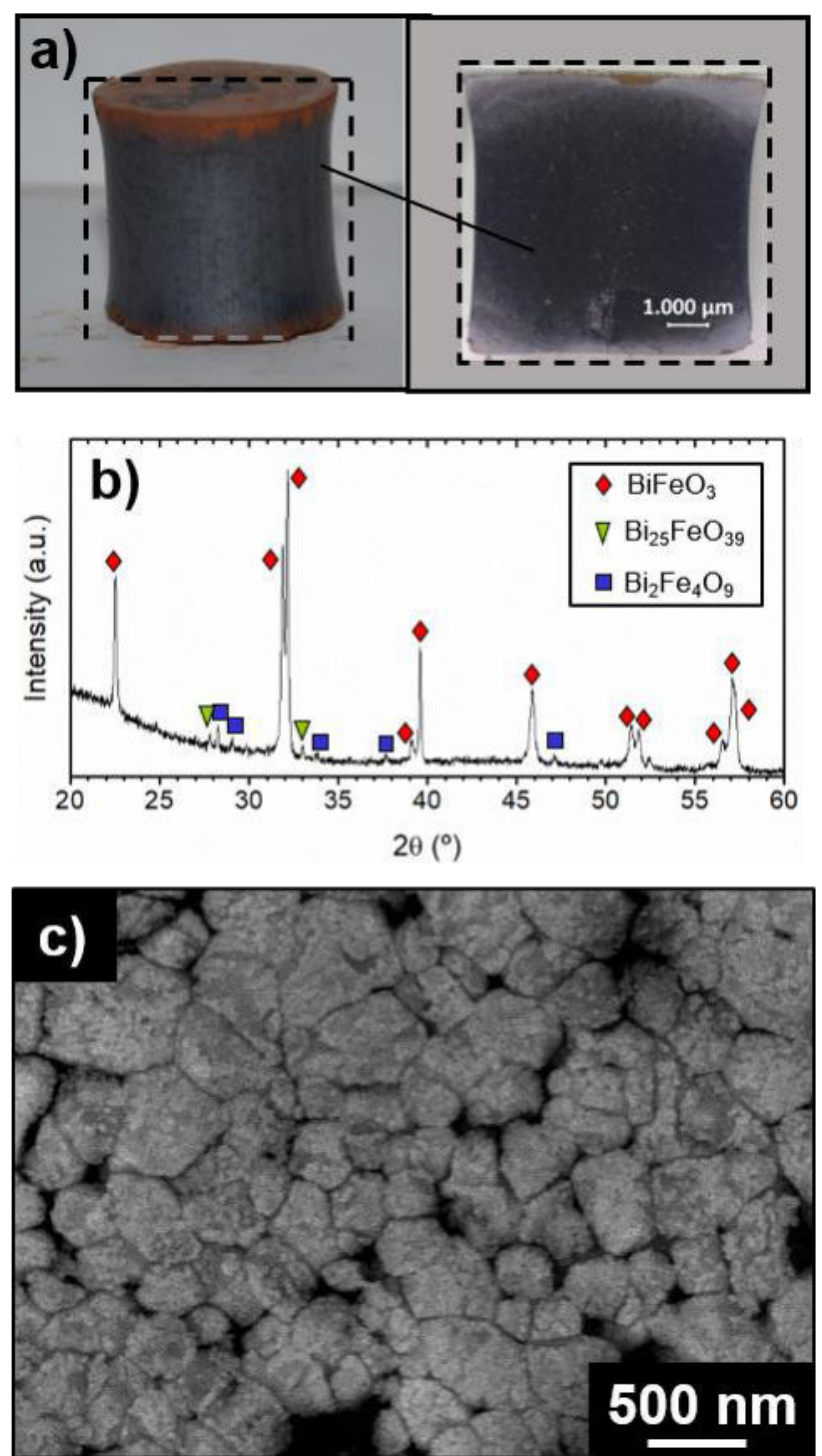

Figure 6. Structural/compositional characterization of the BFTO sample sintered under these 40 V/cm AC conditions: photographs on an entire and a bisected specimen (a), XRD on the polished cross-section surface (b) and scanning electron micrographs on the chemically etched polished cross-section surface $(\mathbf{c})$.

\section{Conclusions:}

Sintering $\mathrm{BiFeO}_{3}$ ceramic powders under a direct current electric field of 50 or $40 \mathrm{~V} / \mathrm{cm}$ results in the development of heterogeneous microstructures related to the current localisation and to thermal gradients within the specimens during the sintering cycles. The formation of macroscopic defects in the flash sintering is prevented if the sintering is carried out under an alternate current electric field of $40 \mathrm{~V} / \mathrm{cm}$, conditions that lead to symmetrical, highly homogeneous microstructures within the entire specimen. The lower 
effective current flowing through the specimens during the AC assisted sintering as well as the larger contribution of the $\mathrm{BiFeO}_{3}$ grain interior conductivity are behind the observed improvement in the microstructural homogeneity. A similar scenario is found for the Ti-doped $\mathrm{BiFeO}_{3}$ composition (BFTO), which also behaves better -from the point of view of microstructure development- under the AC electrical conditions. The presence of titanium slows down the diffusion processes, delaying the onset of the flash event; but a homogeneous microstructure is again observed when applying the flash sintering methodology to this doped formulation under the optimised conditions here described. Furthermore, in this case the flash-sintered specimens show the characteristic nanostructure that is usually developed in the bismuth ferrite materials sintered in presence of titanium, where the submicronic grains are divided in internal domains of nanometric size. Thus, undoped and Ti-doped $\mathrm{BiFeO}_{3}$ bulk materials with functional micro or nanoestructures, which are indeed homogenous at the macroscopic level, can be attained by a flash sintering methodology with lower temperatures and shorter times than by a solid-state conventional sintering method.

\section{Acknowledgements}

This work has been conducted within the MAT2016-80182-R and MAT2014-59210-JIN projects. M.S. Bernardo also acknowledges to the JECS Trust for the financial support for her stay in Jülich, in the frame of the contract 2016118.

\section{References:}

1. Ederer, C. and N.A. Spaldin, A new route to magnetic ferroelectrics. Nature Materials, 2004. 3(12): p. 849-851.

2. Spaldin, N.A. and M. Fiebig, The renaissance of magnetoelectric multiferroics. Science, 2005. 309(5733): p. 391-392.

3. Bibes, M. and A. Barthélémy, Multiferroics: Towards a magnetoelectric memory. Nature Materials, 2008. 7(6): p. 425-426.

4. Catalan, G. and J.F. Scott, Physics and applications of bismuth ferrite. Advanced Materials, 2009. 21(24): p. 2463-2485.

5. Rojac, T., et al., $\mathrm{BiFeO}_{3}$ Ceramics: Processing, electrical, and electromechanical properties. Journal of the American Ceramic Society, 2014.

6. Shi, X.X., X.Q. Liu, and X.M. Chen, Readdressing of Magnetoelectric Effect in Bulk BiFeO3. Advanced Functional Materials, 2017.

7. Qi, X., et al., Greatly reduced leakage current and conduction mechanism in aliovalention-doped $\mathrm{BiFeO}_{3}$. Applied Physics Letters, 2005. 86(6): p. 1-3. 
8. Béa, $\mathrm{H}$. , et al., Influence of parasitic phases on the properties of $\mathrm{BiFeO}_{3}$ epitaxial thin films. Applied Physics Letters, 2005. 87(7): p. 072508.

9. Selbach, S.M., M.A. Einarsrud, and T. Grande, On the Thermodynamic Stability of $\mathrm{BiFeO}_{3}$. Chemistry of Materials, 2009. 21(1): p. 169-173.

10. Bernardo, M.S., et al., Reaction pathways in the solid state synthesis of multiferroic $\mathrm{BiFeO}_{3}$. Journal of the European Ceramic Society, 2011. 31(16): p. 3047-3053.

11. Sosnowska, I., T.P. Neumaier, and E. Steichele, Spiral magnetic ordering in bismuth ferrite. Journal of Physics C: Solid State Physics, 1982. 15(23): p. 4835-4846.

12. Huang, F., et al., Peculiar magnetism of $\mathrm{BiFeO}_{3}$ nanoparticles with size approaching the period of the spiral spin structure. Scientific Reports, 2013. 3.

13. Khomchenko, V.A., et al., Weak ferromagnetism in diamagnetically-doped $\mathrm{Bi}_{1-x} \mathrm{~A}_{x} \mathrm{FeO}_{3}$ $(A=C a, S r, P b, B a)$ multiferroics. Materials Letters, 2008. 62(12-13): p. 1927-1929.

14. Khomchenko, V.A., L.C.J. Pereira, and J.A. Paixão, Weak ferromagnetism and nanodimensional ferroelectric domain structure stabilized in the polar phase of $\mathrm{Bi}_{1-}$ ${ }_{x} \mathrm{Nd}_{x} \mathrm{FeO}_{3}$ multiferroics via Ti doping. Journal of Applied Physics, 2014. 115(16).

15. Bernardo, M.S., T. Jardiel, and M. Villegas, Synthesis and microstructural evolution of $\mathrm{BiFeO}_{3}$ ceramics modified with ZnO. Boletin De La Sociedad Española De Ceramica Y Vidrio, 2010. 49(1): p. 47-52.

16. Bernardo, M.S., et al., Metastable nature of donor-doped BiFeO3 obtained by mechanochemical synthesis. Nippon Seramikkusu Kyokai Gakujutsu Ronbunshi/Journal of the Ceramic Society of Japan, 2016. 124(1): p. 92-97.

17. Bernardo, M.S., Synthesis, microstructure and properties of $\mathrm{BiFeO}_{3}$-based multiferroic materials: A review. Boletin de la Sociedad Espanola de Ceramica y Vidrio, 2014. 53(1): p. 1-14.

18. Marco, C., R. Boriana, and R. Rishi, Flash Sintering of Nanograin Zirconia in $<5 \mathrm{~s}$ at $850^{\circ} \mathrm{C}$. Journal of the American Ceramic Society, 2010. 93(11): p. 3556-3559.

19. Todd, R.I., et al., Electrical characteristics of flash sintering: thermal runaway of Joule heating. Journal of the European Ceramic Society, 2015. 35(6): p. 1865-1877.

20. Guillon, O., et al., Field-assisted sintering technology/spark plasma sintering: Mechanisms, materials, and technology developments. Advanced Engineering Materials, 2014. 16(7): p. 830-849.

21. Yu, M., et al., Review of flash sintering: materials, mechanisms and modelling. Advances in Applied Ceramics, 2017. 116(1): p. 24-60.

22. Gil-Gonzalez, E., et al., Phase-pure BiFeO3 produced by reaction flash-sintering of $\mathrm{Bi}_{2} \mathrm{O}_{3}$ and $\mathrm{Fe}_{2} \mathrm{O}_{3}$. Journal of Materials Chemistry A, 2018. 6(13): p. 5356-5366.

23. Perez-Maqueda, L.A., et al., Flash sintering of highly insulating nanostructured phasepure $\mathrm{BiFeO}_{3}$. Journal of the American Ceramic Society, 2017. 100(8): p. 3365-3369.

24. M'Peko, J.C., J.S.C. Francis, and R. Raj, Field-assisted sintering of undoped BaTiO3: Microstructure evolution and dielectric permittivity. Journal of the European Ceramic Society, 2014. 34(15): p. 3655-3660.

25. Zhang, Y., J.I. Jung, and J. Luo, Thermal runaway, flash sintering and asymmetrical microstructural development of $\mathrm{ZnO}$ and $\mathrm{ZnO}-\mathrm{Bi}_{2} \mathrm{O}_{3}$ under direct currents. Acta Materialia, 2015. 94: p. 87-100.

26. Kim, S.W., et al., Enhanced grain boundary mobility in yttria-stabilized cubic zirconia under an electric current. Journal of the American Ceramic Society, 2011. 94(12): p. 4231-4238.

27. Kim, S.W., S.J.L. Kang, and I.W. Chen, Electro-sintering of yttria-stabilized cubic zirconia. Journal of the American Ceramic Society, 2013. 96(5): p. 1398-1406.

28. Conrad, H. and J. Wang, Equivalence of $A C$ and DC electric field on retarding grain growth in yttria-stabilized zirconia. Scripta Materialia, 2014. 72-73: p. 33-34. 
29. Qin, W., et al., Temperature gradient and microstructure evolution in AC flash sintering of 3 mol\% yttria-stabilized zirconia. Materials and Manufacturing Processes, 2017. 32(5): p. 549-556.

30. Khomchenko, V.A., et al., Doping strategies for increased performance in $\mathrm{BiFeO}_{3}$. Journal of Magnetism and Magnetic Materials, 2009. 321(11): p. 1692-1698.

31. Yang, C.H., et al., Doping BiFeO $\mathrm{O}_{3}$ : Approaches and enhanced functionality. Physical Chemistry Chemical Physics, 2012. 14(46): p. 15953-15962.

32. Khomchenko, V.A., et al., Effect of diamagnetic $\mathrm{Ca}, \mathrm{Sr}, \mathrm{Pb}$, and $\mathrm{Ba}$ substitution on the crystal structure and multiferroic properties of the $\mathrm{BiFeO}_{3}$ perovskite. Journal of Applied Physics, 2008. 103(2): p. 024105.

33. Khomchenko, V.A., et al., Effect of Gd substitution on the crystal structure and multiferroic properties of BiFeO3. Acta Materialia, 2009. 57(17): p. 5137-5145.

34. Bernardo, M.S., et al., Sintering and microstuctural characterization of $\mathrm{W}^{6+}, \mathrm{Nb}^{5+}$ and $\mathrm{Ti}^{4+}$ iron-substituted $\mathrm{BiFeO}_{3}$. Journal of Alloys and Compounds, 2011. 509(26): p. 72907296.

35. Gumiel, $\mathrm{C}$., et al., Thin film composites in the $\mathrm{BiFeO}_{3}-\mathrm{Bi}_{4} \mathrm{Ti}_{3} \mathrm{O}_{12}$ system obtained by an aqueous solution-gel deposition methodology. Boletín de la Sociedad Española de Cerámica y Vidrio, 2018. 57(1): p. 19-28.

36. Liu, H., Z. Liu, and K. Yao, Improved electric properties in $\mathrm{BiFeO}_{3}$ films by the doping of Ti. Journal of Sol-Gel Science and Technology, 2007. 41(2): p. 123-128.

37. $\mathrm{Gu}, \mathrm{Y} . \mathrm{H} .$, et al., Nonstoichiometric $\mathrm{BiFe}_{0.9} \mathrm{Ti}_{0.05} \mathrm{O}_{3}$ multiferroic ceramics with ultrahigh electrical resistivity. Journal of Applied Physics, 2010. 108(9): p. 094112.

38. Kim, S.J., et al., Multiferroic properties of Ti-doped $\mathrm{BiFeO}_{3}$ ceramics. Journal of the Korean Physical Society, 2010. 56(12): p. 439-442.

39. Kalantari, $\mathrm{K}$., et al., Ti-doping to reduce conductivity in $\mathrm{Bi} 0.85 \mathrm{Nd} 0.15 \mathrm{FeO} 3$ ceramics. Advanced Functional Materials, 2011. 21(19): p. 3737-3743.

40. Bernardo, M.S., et al., Intrinsic compositional inhomogeneities in bulk Ti-doped $\mathrm{BiFeO}_{3}$ : Microstructure development and multiferroic properties. Chemistry of Materials, 2013. 25(9): p. 1533-1541.

41. Khomchenko, V.A. and J.A. Paixão, Ti doping-driven magnetic and morphological changes in multiferroic ceramics of $\mathrm{Bi}_{0.9 \mathrm{~L}} \mathrm{La}_{0.1} \mathrm{FeO}_{3}$. Journal of Physics D: Applied Physics, 2015. 48(34).

42. Zuo, R., E. Aulbach, and J. Rödel, Experimental determination of sintering stresses and sintering viscosities. Acta Materialia, 2003. 51(15): p. 4563-4574.

43. Gonzalez-Julian, J. and O. Guillon, Effect of Electric Field/Current on Liquid Phase Sintering. Journal of the American Ceramic Society, 2015. 98(7): p. 2018-2027.

44. Bernardo, M.S., Síntesis, microestructura y propiedades de materiales multiferroicos basados en BiFeO3. 2013, Universidad Autónoma de Madrid: Madrid (Spain).

45. Peiteado, $\mathrm{M}$., et al., $\mathrm{Bi}_{2} \mathrm{O}_{3}$ vaporization from $\mathrm{ZnO}$-based varistors. Journal of the European Ceramic Society, 2005. 25(9): p. 1675-1680. 